\title{
Can Induction Chemotherapy before Concurrent Chemoradiation Impact Circumferential Resection Margin Positivity and Survival in Low Rectal Cancers?
}

\author{
Abu Bakar Hafeez Bhatti' ${ }^{1 *}$, Anum Waheed ${ }^{2}$, Aqsa Hafeez ${ }^{2}$, Ali Akbar' ${ }^{1}$, Aamir \\ Ali Syed ${ }^{1}$, Shahid Khattak ${ }^{1}$, Ather Saeed Kazmi ${ }^{3}$
}

\begin{abstract}
Background: Distance from anal verge and abdominoperineal resection are risk factors for circumferential resection margin (CRM) positivity in rectal cancer. Induction chemotherapy (IC) before concurrent chemoradiation (CRT) has emerged as a new treatment modification. Impact of IC before concurrent CRT on CRM positivity in low rectal cancer remains to be independently studied. The objective of this study was to determine CRM positivity in low rectal cancer, with and without prior IC, and to identify predictors of disease free and overall survival. Materials and Methods: Patients who underwent surgery for rectal cancer between 2005 and 2011 were retrospectively reviewed and divided into two groups. Group 1 received IC before CRT and Group 2 did not. Demographics, clinicopathological variables and CRM status were compared. Actuarial 5 year disease free survival (DFS), overall survival (OS) and independent predictors of survival were determined. Results: Patients in the IC group presented with advanced stage (Stage $3=89.2 \%$ versus $75.4 \%)(\mathrm{P}=\mathbf{0 . 0 2}$ ) but a high rate of total mesorectal excision (TME) $(100 \%$ versus $93.4 \%)(P=0.01)$ and sphincter preservation surgery (54.9\% versus $22.9 \%)(\mathrm{P}=\mathbf{0 . 0 0 1})$. Patients with low rectal cancer who received IC had a significantly low positive CRM rate $(9.2 \%$ versus $34 \%)$ (P=0.002). Actuarial 5 year DFS in IC and no IC groups were $39 \%$ and $43 \%$ $(P=0.9)$ and 5 year $O S$ were $70 \%$ and $47 \%(P=0.003)$. Pathological tumor size [HR: 2.2, CI: 1.1-4.5, $P=0.01]$ and nodal involvement [HR: 2 , CI: $1.08-4, P=0.02]$ were independent predictors of relapse while pathological nodal involvement [HR: 2.6, CI: 1.3-4.9, P=0.003] and IC [HR: 0.7, CI: 0.5-0.9, P=0.02] were independent predictors of death. Conclusions: In low rectal cancer, induction chemotherapy before CRT may significantly decrease CRM positivity and improve 5 year overall survival.
\end{abstract}

Keywords: Circumferential resection margin - rectal cancer - induction chemotherapy - survival

Asian Pac J Cancer Prev, 16 (7), 2993-2998

\section{Introduction}

Low rectal cancer is more prevalent in South East Asia than in the West (Wijenayake et al., 2011). It is more aggressive than upper and mid rectal tumors and is associated with a high local recurrence rate (Fujita et al., 2003; Ueno et al., 2005; Shahib et al., 2010; Akbar et al., 2014). Many of these patients undergo abdominoperineal resection (APR) which further compromises outcomes. AttribuTable factors include high rates of circumferential resection margin (CRM) positivity, inadvertent bowel perforations and substandard mesorectal specimens with APR (den Dulk et al., 2007; 2009). Traditional APR creates a surgical waste between 35 to $42 \mathrm{~mm}$ from anal verge that potentially leads to inferior outcomes (Marr et al., 2005; Shihab et al., 2010).

CRM positivity is an independent predictor of local recurrence, distant metastasis and survival (Quirke et al.,
1986; Birbeck et al., 2002; Wibe et al., 2002). It remains unclear if preoperative chemoradiation alters CRM status (Gosens et al., 2007; Smith et al., 2010; Rullier et al., 2013). Preoperative chemoradiation reduces locoregional recurrence but does not alter systemic relapse which is the most common mode of failure in these patients (Gao et al., 2014). Various induction regimens have been developed and administered before chemoradiation in an attempt to reduce distant failure but equivocal outcomes have been reported (Chau et al., 2006; Fernandez Martos et al., 2010; Calvo et al., 2014).

A recently concluded study showed improved pathological complete response (PCR) and R0 rates with induction but failed to show any long term improvement in outcomes (Calvo et al., 2014). The impact of induction chemotherapy before concurrent chemoradiation on CRM positivity rate in low rectal cancer has not been studied independently. The objective of this study was to compare 
CRM positivity in low rectal cancer between patients who received chemoradiation after induction chemotherapy versus chemoradiation alone. Secondarily, we also looked at independent predictors of disease free and overall survival in these patients.

\section{Materials and Methods}

A retrospective review of patients who underwent surgery for rectal adenocarcinoma between January 2005 and December 2011 at Shaukat Khanum Memorial Cancer Hospital and Research Centre was performed. A total of 154 patients who underwent surgical resection with curative intent and received preoperative chemoradiotherapy were included. Patients who underwent palliative surgery and patients in whom circumferential resection margin status (CRM) could not be determined were excluded.

Standard workup for these patients included clinical exam, endoscopy, MRI pelvis and CT scan chest and abdomen. We staged patients according to American Joint Committee on Cancer (AJCC) 7th staging manual. Treatment was initiated after a plan was formulated in weekly multidisciplinary team meeting. The standard protocol was to administer preoperative chemoradiotherapy in T3/T4 and/ or clinically node positive tumors. Use of induction chemotherapy was on discretion of treating medical oncologist. The standard induction chemotherapy regimen consisted of 4 cycles of Capecitabine and Oxaliplatinum 3 weeks apart. Each cycle consisted of Capecitabine $1000 \mathrm{mg} / \mathrm{m} 2$ oral twice daily on Day 1 and 14 and Oxaliplatinum $130 \mathrm{mg} / \mathrm{m} 2$ intravenous on Day 1. In the concurrent setting, 825 $\mathrm{mg} / \mathrm{m} 2$ of Capecitabine was given orally twice daily continuously throughout radiation. A total of $50.4 \mathrm{~Gy}$ of radiation was administered in 28 fractions with 1.8 Gy per fraction, 5 days a week over 5 and a half weeks. After completion of neoadjuvant treatment, response assessment was performed with MRI pelvis and CT scan chest and abdomen. Surgery was usually performed in 6-8 weeks. Standard principles of total mesorectal excision (TME) were applied during proctectomy. Both open and laparoscopic approaches were used. Patients were followed 3 monthly for 1 year, 6 monthly for 2 years and then yearly onwards with Carcinoembryonic antigen (CEA) levels. CT scan and endoscopy was performed yearly.

Patient demographics and clinicopathological variables were compared. Patients were divided into two groups. Group 1 received induction chemotherapy(IC) and Group 2 did not (No IC). Low rectal cancers were defined as tumors within $5 \mathrm{~cm}$ from anal verge. The primary outcome of interest was circumferential resection margin (CRM) positivity rate in both groups. A CRM $\leq 1 \mathrm{~mm}$ was considered positive while $>1 \mathrm{~mm}$ was labelled negative in the current study. Adverse events and 5 year actuarial disease free survival (DFS) and overall survival (OS) was determined for low rectal cancers and compared based on whether they did or did not receive IC. Disease free survival was defined as time between date of surgery and date of relapse. Relapse included local, regional and distant failures. Overall survival was defined as time between date of surgery and date of death or last follow-up. Categorical variables were compared with chi square test and Fischer's exact test. For interval variables, t test and Mann-Whitney U test were used.Actuarial 5 year survival was calculated using Kaplan Meier survival curves and Log rank test was used to determine significance. A P value $<0.05$ was considered significant for all calculations. A multivariate Cox proportional hazard model was used to determine independent predictors of outcome. Specifically we included age group $(<40$ versus $>40)$, gender, clinical tumor stage (cT), clinical nodal stage $(\mathrm{cN})$, pathological tumor stage $(\mathrm{pT})$, pathological nodal stage $(\mathrm{pN})$, circumferential resection margin status (CRM), total mesorectal excision (TME versus no TME), surgical procedure (APR versus others), IC/No IC and pathological

Table 1. Patient Characteristics

\begin{tabular}{|c|c|c|c|c|c|c|}
\hline & & \multicolumn{2}{|c|}{ IC given } & \multicolumn{2}{|c|}{ No IC given } & \multirow[t]{2}{*}{$\mathrm{P}$ value } \\
\hline & & Number $\mathrm{N}=93$ & Percent & Number $\mathrm{N}=61$ & Percent & \\
\hline Gender & Male & 63 & 67.7 & 42 & 68.9 & 0.8 \\
\hline Age group & Less than 40 & 49 & 52.6 & 27 & 44.2 & 0.3 \\
\hline Family History & Positive & 6 & 6.5 & 0 & 0 & $0.01 *$ \\
\hline \multirow[t]{3}{*}{ Clinical tumor stage } & $\mathrm{T} 2$ & 11 & 11.8 & 4 & 6.5 & 0.2 \\
\hline & $\mathrm{T} 3$ & 56 & 60.2 & 45 & 73.8 & \\
\hline & $\mathrm{T} 4$ & 26 & 28 & 12 & 19.7 & \\
\hline \multirow[t]{3}{*}{ Clinical nodal stage } & NO & 9 & 9.7 & 15 & 24.6 & $0.001 *$ \\
\hline & N1 & 13 & 14 & 17 & 27.9 & \\
\hline & $\mathrm{N} 2$ & 71 & 76.3 & 29 & 47.5 & \\
\hline \multirow[t]{2}{*}{ Overall Stage } & 2 & 10 & 10.8 & 15 & 24.6 & $0.02 *$ \\
\hline & 3 & 83 & 89.2 & 46 & 75.4 & \\
\hline \multirow[t]{2}{*}{ Distance from anal verge } & $<5 \mathrm{~cm}$ & 65 & 69.9 & 47 & 77 & 0.4 \\
\hline & $>5 \mathrm{~cm}$ & 28 & 30.1 & 14 & 27 & \\
\hline \multirow[t]{4}{*}{ Procedure } & Others & 2 & 2.2 & 2 & 3.3 & $0.001 *$ \\
\hline & APR & 40 & 43 & 45 & 73.8 & \\
\hline & LAR & 37 & 39.8 & 13 & 21.3 & \\
\hline & ULAR & 14 & 15.1 & 1 & 1.6 & \\
\hline \multirow[t]{2}{*}{ Approach } & Laparoscopic & 29 & 31.1 & 24 & 39.3 & 0.3 \\
\hline & Open & 64 & 68.9 & 37 & 60.7 & \\
\hline
\end{tabular}

IC: induction chemotherapy; APR: abdominoperineal resection; LAR: low anterior resection; ULAR: ultralow anterior resection; *p<0.05 
complete response (PCR) for univariate analysis. Variables significant on univariate analysis were included in multivariate model to determine independent predictors of outcome. The study was performed in accordance with declaration of Helsinki and the hospital ethics committee granted exemption from formal review of this study.

\section{Results}

\section{Patient characteristics}

Minimum follow-up was 28 months. Median follow up was 45(7.2-108) months. Median age was 41years (15$72)$. Male to female ratio was $2: 1(105 / 154$ versus 49/154). A total of 93 patients received induction chemotherapy (IC) before chemoradiation while 61 patients only received chemoradiation. Table 1 represents clinical characteristics of patients who received IC versus those who did not. Significant difference was present between the two groups with respect to family history and clinical stage of tumor. In the IC group, 6(6.5\%) patients had a positive family history versus 0 patients in No-IC group $(\mathrm{p}=0.01)$. More patients in IC group (76) (76.3\%) versus No-IC group (29) (47.5\%) had N2 disease ( $\mathrm{p}=0.001)$. Abdominoperineal resection (APR) was more frequently performed in patients who did not receive IC 45(73.8\%) versus $40(43 \%)(\mathrm{p}=0.001)$. Table 2 represents the toxicity associated with induction chemotherapy in patients with rectal carcinoma. Neurological toxicity was most common and seen in 18(19.3\%) patients who received IC followed by gastrointestinal toxicity in $15(16 \%)$ patients. Overall $13(13.9 \%)$ patients had grade III toxicity.

\section{Histopathological variables}

No significant difference in pathological tumor and nodal stage was observed. More patients in the IC group had a complete pathological response (PCR) (30.1\%) versus $(19.7 \%)$ but it did not reach significance $(\mathrm{p}=0.1)$. No significant difference was observed in the mean number of lymph nodes removed and mean number of positive nodes between two groups as shown in Table 3 . Total mesorectal excision was performed in all 93(100\%) patients who received IC versus 57(93.4\%) patients in No-IC group $(\mathrm{p}=0.01)$.

\section{CRM status}

Out of total 93 patients who received IC, 85(91.4\%) had a negative CRM versus $44 / 61(72.1 \%$ ) patients in the No-IC group ( $\mathrm{p}=0.002)$. On subgroup analysis, $88 \%$ of CRM positive patients had low rectal tumors. The CRM positivity in low rectal tumors was $9.2 \%$ and $34 \%$ in IC and No IC groups respectively ( $\mathrm{p}=0.002)$. Most importantly patients who received induction chemotherapy had no difference in CRM positivity rate in high versus low rectal tumors i.e. $2 / 28(7.1 \%$ ) versus $6 / 65(9.2)(\mathrm{p}=0.9)$. There was a significant difference between low and high rectal tumors in the No IC group in terms of CRM positivity $1 / 14(7.1 \%)$ versus $16 / 47(34 \%)(\mathrm{p}=0.04)$ as shown in Table 4.

\section{Outcomes in low rectal cancer}

We looked specifically on pattern of relapse in low rectal cancers. There were 28 relapses in IC group versus 23 in No IC group ( $p=0.5)$. Although locoregional relapses were more frequent in No IC group, the difference did not

Table 2. Toxicity Profile in Patients who Received Induction Chemotherapy

\begin{tabular}{lccccccc}
\hline & $\begin{array}{c}\text { Gastrointestinal } \\
\mathrm{N}=15\end{array}$ & $\begin{array}{c}\text { Neurological } \\
\mathrm{N}=18\end{array}$ & $\begin{array}{c}\text { Myelosuppression } \\
\mathrm{N}=2\end{array}$ & $\begin{array}{c}\text { Others } \\
\mathrm{N}=1\end{array}$ & $\begin{array}{c}\text { Total } \\
\text { N=93 }\end{array}$ & Percent \\
\hline Toxicity & Grade 1 & 1 & 8 & 0 & 0 & 9 & 9.6 \\
& Grade 2 & 3 & 10 & 0 & 1 & 15 & 16.1 \\
& Grade 3 & 11 & 0 & 2 & 0 & 13 & 13.9 \\
\hline
\end{tabular}

Table 3. Histopathological Variables in the two Groups

\begin{tabular}{|c|c|c|c|c|c|c|}
\hline & & \multicolumn{2}{|c|}{ IC given } & \multicolumn{2}{|c|}{ No IC given } & \multirow[t]{2}{*}{$\mathrm{P}$ value } \\
\hline & & Number $\mathrm{N}=93$ & Percent & Number $\mathrm{N}=61$ & Percent & \\
\hline \multirow[t]{3}{*}{ Grade } & Well & 21 & 22.6 & 10 & 16.4 & 0.1 \\
\hline & Moderate & 50 & 53.8 & 41 & 67.2 & \\
\hline & Poor & 22 & 23.6 & 10 & 16.4 & \\
\hline \multirow[t]{5}{*}{ Pathological tumor stage } & 0 & 29 & 31.2 & 13 & 21.3 & 0.4 \\
\hline & 1 & 6 & 6.5 & 2 & 3.3 & \\
\hline & 2 & 15 & 16.1 & 9 & 14.8 & \\
\hline & 3 & 37 & 39.8 & 33 & 54.1 & \\
\hline & 4 & 6 & 6.5 & 4 & 6.6 & \\
\hline \multirow[t]{3}{*}{ Pathological nodal stage } & No & 60 & 64.5 & 31 & 50.8 & 0.1 \\
\hline & N1 & 16 & 17.2 & 10 & 16.4 & \\
\hline & $\mathrm{N} 2$ & 17 & 18.3 & 20 & 32.8 & \\
\hline Lymphovascular invasion & Present & 11 & 11.8 & 4 & 6.6 & 0.1 \\
\hline Pathological complete response & Present & 28 & 30.1 & 12 & 19.7 & 0.1 \\
\hline \multirow[t]{2}{*}{ Total mesorectal excision } & Performed & 93 & 100 & 57 & 93.4 & $0.01 *$ \\
\hline & & Mean & SD & Mean & $\mathrm{SD}$ & \\
\hline Examined nodes & & 12.3 & 4.6 & 11.5 & 5.3 & 0.3 \\
\hline Positive nodes & & 1.7 & 3.5 & 2.8 & 4 & 0.07 \\
\hline Distal margin(mm) & & 33 & 26.7 & 33.6 & 19.8 & 0.8 \\
\hline
\end{tabular}

IC induction chemotherapy; ${ }^{*} \mathrm{p}<0.05$ 
Table 4. CRM Status in Patients Who Received Induction Chemotherapy Versus Patients who did not

\begin{tabular}{|c|c|c|c|c|}
\hline & & $\begin{array}{c}\text { IC given } \\
\text { No. }(\%) \\
N=93\end{array}$ & $\begin{array}{c}\text { No IC give } \\
\text { No. }(\%) \\
\mathrm{N}=61\end{array}$ & en value \\
\hline Negative CRM & $>1 \mathrm{~mm}$ & $85(91.4)$ & $44(72.1)$ & $0.002^{*}$ \\
\hline Positive CRM & $<1 \mathrm{~mm}$ & $\begin{aligned} 8(8.6) \\
\mathrm{N}\end{aligned}$ & $\begin{array}{c}17(27.9) \\
\mathrm{N}=47\end{array}$ & \\
\hline$<5 \mathrm{~cm}$ from anal verge & $<1 \mathrm{~mm}$ & $\begin{aligned} 6 & (9.2) \\
\mathrm{N} & =28\end{aligned}$ & $\begin{array}{c}16(34) \\
N=14\end{array}$ & $0.002 *$ \\
\hline$>5 \mathrm{~cm}$ from anal verge & $<1 \mathrm{~mm}$ & $2(7.1)$ & $1(7)$ & 0.9 \\
\hline
\end{tabular}

Table 5. Pattern of Locoregional and Distant Relapses in Patients with Low Rectal Cancer who Received IC or notb IC Induction Chemotherapy

\begin{tabular}{|c|c|c|c|c|c|}
\hline & \multicolumn{2}{|c|}{$\begin{array}{l}\text { IC group in low } \\
\text { rectal tumors } \\
\quad \mathrm{N}=65\end{array}$} & \multicolumn{2}{|c|}{$\begin{array}{l}\text { No IC group in low } \\
\text { rectal tumors } \\
\mathrm{N}=47\end{array}$} & \multirow[t]{2}{*}{$P$ value } \\
\hline & Number & Percent & Number & Percent & \\
\hline Total & 28 & 43 & 23 & 48.9 & 0.5 \\
\hline Locoregional & 12 & 18.4 & 11 & 23.4 & 0.6 \\
\hline Distant & 16 & 24.6 & 12 & 25.5 & 1 \\
\hline
\end{tabular}

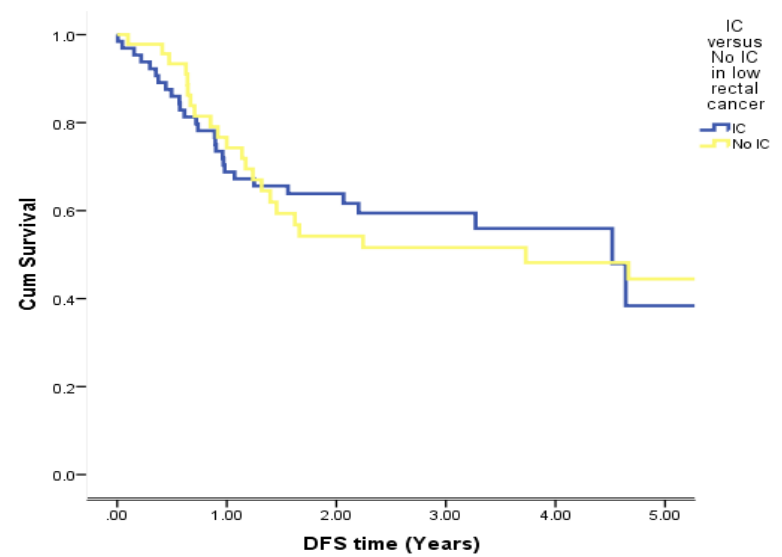

Figure 1. Actuarial 5 year Disease free Survival in Patients with Low Rectal Cancer who Received Induction Chemotherapy or Not, $39 \%$ versus $43 \%$, $\mathbf{p}=0.9$

Table 6. Multivariate Cox Proportional Hazard Model for Independent Predictors of Relapse and Death

\begin{tabular}{|c|c|c|c|}
\hline & $\begin{array}{r}\mathrm{R} \\
\text { Multiva }\end{array}$ & $\begin{array}{l}\text { se } \\
\text { analysis }\end{array}$ & \\
\hline & $\begin{array}{c}\text { Hazard } \\
\text { ratio }\end{array}$ & $\begin{array}{c}\text { Confidence } \\
\text { interval }\end{array}$ & $P$ value \\
\hline Pathological tumor s & & & \\
\hline pT1T2 & 1 & $1.1-4.5$ & 0.01 \\
\hline pT3T4 & 2.29 & & \\
\hline Pathological nodal in & olvemen & & \\
\hline No & 1 & $1.08-4$ & 0.02 \\
\hline $\mathrm{N}+$ & 2.08 & & \\
\hline Death & & & \\
\hline Pathological nodal & ivolveme & & \\
\hline No & 1 & $1.3-4.9$ & 0.003 \\
\hline $\mathrm{N}+$ & 2.6 & & \\
\hline Induction chemothe & apy & & \\
\hline Not given & 1 & $0.5-0.9$ & 0.02 \\
\hline given & 0.7 & & \\
\hline
\end{tabular}

Multivariate Cox proportional hazard model for independent predictors of relapse and death

2996 Asian Pacific Journal of Cancer Prevention, Vol 15, 2014



Figure 2. Actuarial 5 year Overall Survival in Patients with Low Rectal Cancer who Received Induction Chemotherapy or Not, $70 \%$ and $47 \%(p=0.003)$

reach significance $(\mathrm{p}=0.6)$ (Table 5). Median DFS in IC and No IC group was $1.8(0.01-5.2)$ years and 1.5(0.1-6.6) years $(\mathrm{p}=0.6)$. Median OS in two groups was 3.5(0.9-7.4) years and $3.2(0.6-9)$ years $(\mathrm{p}=1)$. Actuarial 5 year DFS in IC and No IC groups was $39 \%$ and $43 \%(\mathrm{p}=0.9)$. Actuarial 5 year OS was $70 \%$ and $47 \%(\mathrm{p}=0.003)$ respectively in the two groups and was significantly different as shown in Figure 1. Table 6 demonstrates independent predictors of relapse and death. On univariate analysis for relapse, grade $(\mathrm{p}=0.04), \mathrm{pT}(\mathrm{p}=0.002) \mathrm{pN}(\mathrm{p}=0.0001), \mathrm{TME}(\mathrm{p}=0.02)$ and PCR $(p=0.002)$ were significant variables. On multivariate analysis however, pathological tumor size and nodal involvement were independent predictors of relapse. Both T3T4 tumors and nodal involvement on histopathology doubled the risk of relapse as shown in Table 6. On univariate analysis, pT (0.01), pN (0.001), CRM (0.0080, TME (0.01), PCR (0.008) and IC (0.004) were significant variables for risk of death. On multivariate analysis, nodal involvement on histopathology increased risk of death (HR: 2.6, CI: 1.3-4.9, $\mathrm{p}=0.003$ ) while use of IC reduced the risk of death by $30 \%$ (HR: $0.7, \mathrm{CI}: 0.5-0.9, \mathrm{p}=0.02$ ).

\section{Discussion}

The current study demonstrates plausible role of induction chemotherapy (IC) before concurrent chemoradiation in low rectal cancers. No difference in CRM positivity between the two groups was observed for tumors located $>5 \mathrm{~cm}$ from anal verge. Majority of patients however had low rectal tumors and without prior IC, CRM positivity was significantly high. At presentation, our patients had more advanced tumor size and nodal stage when compared with other studies. A median follow up of 45 months was sufficiently long in comparison with previous studies. Induction chemotherapy significantly improved 5 year overall survival in low rectal cancers despite comparable disease free survival.

Neoadjuvant chemoradiation followed by TME is the current standard of care for locally advanced on node positive rectal cancer (Zeng et al., 2014). After preoperative chemoradiation, positive CRM rate can vary between 3-14\% (Fernandez-Martos et al., 2010; Kellokumpu et al., 2012; Kenelley et al., 2013; Rullier et al., 2013; Calvo et al., 2014). CRM positivity as high as $26 \%$ has been reported in patients with low rectal cancers 
who undergo APR (Nagtegaal et al., 2002; 2005; Bernstein et al., 2009). In modern practice, suggested target value of a positive CRM is close to $10 \%$ (Nagtegaal et al., 2002; Wibe et al., 2004). A recent study from Malaysia reported a CRM positive rate of $18.4 \%$ after long course chemoradiation and surgery (Lee et al., 2013). In the current study, CRM positivity was seen in $16.2 \%$ patients and was higher than the current recommendations. On subgroup analysis, it becomes evident that more than $2 / 3 \mathrm{rd}$ of positive CRMs were seen in patients with low rectal tumors without prior IC. Almost all patients in this group underwent APR while sphincter preservation was possible in twice as many patients in the IC group. If we exclude this group from analysis, CRM positivity decreases to $5.8 \%$ which is well below the standard. Several other factors might be responsible for high CRM positivity in No IC group in our study. Although neoadjuvant treatment significantly down staged tumors on final histopathology, significant portion of our patients had pT3/T4 tumors and pN2 nodal disease. It has been shown before that locally advanced pT4 tumors and pathological nodal positivity adversely impact CRM status (Wibe et al., 2004; Kenelly et al., 2013). We generally operate patients within 6-8 weeks of chemoradiation but due to tremendous workload, this was not always possible. Patients with low rectal tumors who underwent APR after a longer than expected duration of 6-8 weeks were more likely to have intense fibrosis thus making identification of surgical planes more difficult. More than $2 / 3$ rd of our patients were males. A narrow male pelvis makes dissection at pelvic diaphragm more demanding and increases CRM positivity (Chapius et al., 2006). In addition, contribution of learning curve to CRM positivity cannot be ruled out as during the study period, there was an increasing effort to perform more procedures laparoscopically. Laparoscopic resection and lap to open converted procedures have been associated with high positive CRM rates (Leonard et al., 2010).

Patients in the IC group experienced several benefits over the other group. Locoregional failures were lower in this group though not statistically significant. Use of induction chemotherapy was an independent predictor of improved survival while pathological nodal involvement was a harbinger of poor outcome. Patients who did not receive IC had a high frequency of $\mathrm{pN}+$ disease though $\mathrm{cN}+$ disease was more frequent in the IC group. We believe use of IC and low frequency of $\mathrm{pN}+$ disease both contributed to significantly improved overall survival in IC group. Sphincter preservation was possible far more frequently in the IC group and a good number of patients underwent ultralow and low anterior resection. The toxicity profile of induction chemotherapy was comparable to a previously conducted phase II study (Fernandez-Martos et al., 2010). Median DFS and OS were not significantly different between the two groups but a trend in favor of IC group was present. Also, actuarial 5 year OS was significantly improved for patients with low rectal cancers who received IC.

Limitations of the current study include its retrospective design and relatively small sample size which prevented matching of significant variables in the two groups. Variability in treatment and selection bias for a particular regimen could not be accounted for due to retrospective nature of the current study. A significant difference was present between IC and No IC groups with respect to clinical nodal stage, overall clinical stage, TME rate and type of surgical procedure (LAR versus APR). Since data in the current study was retrospectively reviewed and was collected based on surgical resection, patients who did not complete induction/concurrent chemoradiation or became irresectable during neoadjuvant treatment could not be identified.

The current study highlights several demographic traits of our distinct population. Younger age, locally advanced disease and high frequency of low rectal tumors was characteristic. In low rectal tumors, IC resulted in significantly improved CRM negative rate when compared with No IC group. Since majority of patients with low rectal cancer without prior IC underwent APR. It is difficult to ascertain if APR or absence of IC was responsible for high CRM positivity. A case can be made for extra levator APR which in multicenter observational study has shown to be superior to standard APR in terms of positive CRM (West et al., 2010). Although IC demonstrated favorable role in terms of sphincter preservation, CRM negativity and overall survival; prospective studies with larger sample size and longer follow up are needed to validate the results of current study.

\section{References}

Akbar A, Bhatti AB, Khattak S, et al (2014). Outcome of rectal cancer in patients aged 30 years or less in the Pakistani population. Asian Pac J Cancer Prev, 15, 6339-42.

Bernstein TE, Endreseth BH, Romundstad P, et al (2009). Circumferential resection margin as a prognostic factor in rectal cancer. Br J Surg, 96, 1348-57.

Birbeck KF, Macklin CP, Tiffin NJ, et al (2002). Rates of circumferential resection margin involvement vary between surgeons and predict outcomes in rectal cancer surgery. Ann Surg, 235, 449-57.

Calvo FA, Sole CV, Serrano J, et al (2014). Preoperative chemoradiation with or without induction oxaliplatin plus 5-fluorouracil in locally advanced rectal cancer. Long-term outcome analysis. Strahlenther Onkol, 190, 149-57.

Chapuis PH, Lin BP, Chan C, Dent OF, Bokey EL (2006). Risk factors for tumour present in a circumferential line of resection after excision of rectal cancer. Br J Surg, 93, 860-5.

Chau I, Brown G, Cunningham D, et al (2006). Neoadjuvant Capecitabine and oxaliplatin followed by synchronous chemoradiation and total mesorectal excision in magnetic resonance imaging defined poor risk rectal cancer. J Clin Oncol, 24, 668-674.

den Dulk M, Marijnen CA, Putter H, et al (2007). Risk factors for adverse outcome in patients with rectal cancer treated with an abdominoperineal resection in the total mesorectal excision trial. Ann Surg, 246, 83-90.

den Dulk M, Putter H, Collette L, et al (2009). The abdominoperineal resection itself is associated with an adverse outcome: the European experience based on a pooled analysis of five European randomised clinical trials on rectal cancer. Eur J Cancer, 45, 1175-83.

Fernandez-Martos C, Pericay C, Aparicio J, et al (2010). Phase II, randomized study of concomitant chemoradiotherapy followed by surgery and adjuvant capecitabine plus oxaliplatin (CAPOX) compared with induction CAPOX 
followed by concomitant chemoradiotherapy and surgery in magnetic resonance imaging-defined, locally advanced rectal cancer: Grupo cancer de recto 3 study. J Clin Oncol, 28, 859-65.

Fernandez Martos C, Pericay C, Aparicio J, et al (2010). Phase II, randomized study of concomitant chemoradiotherapy followed by surgery and adjuvant capecitabine plus oxaliplatin (CAPOX) compared with induction CAPOX followed by concomitant chemoradiotherapy and surgery in magnetic resonance imaging defined, locally advanced rectal cancer: Grupo cancer de recto 3 study. J Clin Oncol, 28, 859-65.

Fujita S, Yamamoto S, Akasu T, Moriya Y (2003). Lateral pelvic lymph node dissection for advanced lower rectal cancer. $\mathrm{Br}$ J Surg, 90, 1580-5.

Gao YH, An X, Sun WJ, et al (2014). Evaluation of capecitabine and oxaliplatin administered prior to and then concomitant to radiotherapy in high risk locally advanced rectal cancer. J Surg Oncol, 109, 478-82.

Gosens MJ, Klaassen RA, Tan-Go I, et al (2007). Circumferential margin involvement is the crucial prognostic factor after multimodality treatment in patients with locally advanced rectal carcinoma. Clin Cancer Res, 13, 6617-23.

Kellokumpu I, Vironen J, Kairaluoma M, et al (2012). Quality of surgical care, local recurrence, and survival in patients with low- and midrectal cancers following multimodal therapy. Int J Colorectal Dis, 27, 111-20.

Kennelly RP, Rogers AC, Winter DC; Abdominoperineal Excision Study Group (2013). Multicentre study of circumferential margin positivity and outcomes following abdominoperineal excision for rectal cancer. Br J Surg, 100, 160-6.

Lee WC, Yusof MM, Lau FN, Phua VC (2013). Preoperative long course chemoirradiation in a developing country for rectal carcinoma: Kuala Lumpur hospital experience. Asian Pac J Cancer Prev, 14, 3941-4.

Leonard D, Penninckx F, Fieuws S, et al (2010). PROCARE, a multidisciplinary Belgian project on cancer of the rectum. Factors predicting the quality of total mesorectal excision for rectal cancer. Ann Surg, 252, 982-8.

Marr R, Birbeck K, Garvican J, et al (2005). The modern abdominoperineal excision: the next challenge after total mesorectal excision. Ann Surg, 242, 740-82.

Nagtegaal ID, Marijnen CA, Kranenbarg EK, et al (2002). Circumferential margin involvement is still an important predictor of local recurrence in rectal carcinoma: not one millimeter but two millimeters is the limit. Am J Surg Pathol, 26, 350-7.

Nagtegaal ID, van de Velde CJ, Marijnen CA, et al (2005). Low rectal cancer: a call for a change of approach in abdominoperineal resection. J Clin Oncol, 23, 9257-64.

Quirke P, Durdey P, Dixon MF, Williams NS (1986). Local recurrence of rectal adenocarcinoma due to inadequate surgical resection. Histopathological study of lateral tumour spread and surgical excision. Lancet, 2, 996-99.

Rullier A, Gourgou-Bourgade S, Jarlier M, et al (2013). Predictive factors of positive circumferential resection margin after radiochemotherapy for rectal cancer: the French randomised trial ACCORD12/0405 PRODIGE 2. Eur J Cancer, 49, 82-9.

Shihab OC, Brown G, Daniels IR, et al (2010). Patients with low rectal cancer treated by abdominoperineal excision have worse tumors and higher involved margin rates compared with patients treated by anterior resection. Dis Colon Rectum, 53, 53-6.

Smith FM, Waldron D, Winter DC, (2010). Rectum-conserving surgery in the era of chemoradiotherapy. Br J Surg, 97,
1752-64.

Ueno M, Oya M, Azekura K, Yamaguchi T, Muto T (2005). Incidence and prognostic significance of lateral lymph node metastasis in patients with advanced low rectal cancer. $\mathrm{Br}$ J Surg, 92, 756-63.

West NP, Anderin C, Smith KJ, et al (2010). Multicentre experience with extralevator abdominoperineal excision for low rectal cancer. Br J Surg, 97, 588-99.

Wibe A, Rendedal PR, Svensson E, et al (2002). Prognostic significance of the circumferential resection margin following total mesorectal excision for rectal cancer. $\mathrm{Br} J$ Surg, 89, 327-34.

Wibe A, Syse A, Andersen E, et al : Norwegian Rectal Cancer Group .(2004). Oncological outcomes after total mesorectal excision for cure for cancer of the lower rectum: anterior vs. abdominoperineal resection. Dis Colon Rectum, 47, 48-58.

Wijenayake W, Perera M, Balawardena J, et al (2011). Proximal and distal rectal cancers differ in curative resectability and local recurrence. World J Gastrointest Surg, 3, 113-8.

Zeng WG, Zhou ZX, Wang Z, et al, (2014). Lymph node ratio is an independent prognostic factor in node positive rectal cancer patients treated with preoperative chemoradiotherapy followed by curative resection. Asian Pac J Cancer Prev, 15, 5365-9. 